\section{Electron densities in active galaxies}

IT is alarming to find a common fallacy concerning the measurement of electron densities in astrophysical conditions appearing twice in the same issue of Nature. Both Fairall ${ }^{1}$ and $\mathrm{Gaske}^{2}$, argue that the mere appearance of the forbidden lines of O III in a Seyfert galaxy can place an upper limit on the electron density and thus a lower limit to the volume of the emitting gas. This simple argument by itself is spurious. To determine the electron density one must either examine the ratio of two lines whose sensitivity to $n_{\mathrm{e}}$ is different, or use a quantitative measurement of a line-to-continuum ratio.

The range of 'typical' electron densities in Seyfert galaxies has been established through proper modelling and it is unfortunate that simplifications of the arguments have led to the two factually incorrect statements which appear, "As the quenching density is $\sim 10^{4} \mathrm{~cm}^{-3}$, we cannot reasonably appeal to higher densities"1 , and "Whenever we see forbidden lines, therefore, we know that the gas density is lower than some critical value"2.

Although well known to many astronomers, it seems useful to outline how the population of the metastable level, from which the forbidden transition occurs, varies as $n_{\mathrm{e}}$ increases. At low densities, collisions between electrons and the lower level excite the upper level and are balanced by spontaneous radiative decay-the 'coronal' approximation. Once the collisional de-excitation rate begins to exceed the spontaneous radiative decay rate the population increases less rapidly, but tends to its maximum value, the Boltzmann population, when collisional excitation and de-excitation balance each other. Thus the intensity of a forbidden line increases as the density increases until the Boltzmann limit is reached. The ratio of the intensity of such a line to that of a permitted line, which has a larger transition probability and hence does not reach its Boltzmann limit until much higher densities, will begin to decrease once the metastable level reaches its maximum population. The behaviour with respect to the continuum depends, of course, on how the latter is formed.

The confusions in the descriptions in the literature may have arisen from an incorrect analogy with 'quenching' in laboratory gases ${ }^{3}$ where different processes may cause the de-excitation and excitation and prevent a true Boltzmann population being reached.

The size of the emitting region in the Seyfert galaxy discussed ${ }^{1}$ is therefore open to question until the relative intensities of the lines or continuum emission present are investigated, but the main point of this comment is to correct the misleading generalizations which have appeared ${ }^{1,2}$.

\section{JORDAN}

Department of Theoretical Physics, Oxford University,

1 Keble Road,

Oxford OX1 3NP, UK 1. Fairall, A. P. Nature 304, 241-243 (1983).
2. Gaskell, C. M. Nature 304, 212-213 (1983).
3. Corney, A. Atomic and Laser Spectroscopy (Oxford
Science Publications, 1977).

FAIRALL REPLIES-The value for the upper limit of the electron density, $n_{\mathrm{e}}=$ $10^{6}$, used in my letter ${ }^{1}$, is simply a standard value widely quoted in the literature (see ref. 2, for example). Whilst I take Jordan's point about the need to determine $n_{\mathrm{e}}$ from sensitive line ratios rather than the appearance of forbidden lines, my value reflects the general consensus and would be acceptable to the experts in the field, one of whom was a referee to my letter.

\section{A. P. FAIrall}

Department of Astronomy,

Rondebosch 7700 ,

Cape, South Africa 1. Fairall, A. P. Nature 304, 241-243 (1983).
2. Tohline, J. E. \& Osterbrock, D. E. Astrophys. J. Leth. 210,
L117 (1976).

GASKELL REPLIES-While recognizing that the main point of Jordan's comment is to attempt to correct the allegedly misleading generalizations made by Fairall $^{1}$ and myself ${ }^{2}$, I must state that the size of the forbidden line emitting region in Fairall 427 is not an open question. If one looks at Fairall's letter ${ }^{1}$ one sees that all the line ratios point to low densities. For example, Fairall gives the ratio of the (forbidden) $[\mathrm{O}$ III] $] \lambda 5,007$ line to the (permitted) $\mathrm{H} \lambda 4,861$ line as 19 . At the same time the $[\mathrm{O}$ III $] \lambda 4,363 /\left[\mathrm{O}_{\mathrm{III}}\right] \lambda 5,007$ ratio, while not explicitly given in the text, is obviously very low (see Fairall's Fig. 1). Quantitative studies of photoionized gases show that such ratios rule out electron densities greater than $10^{6} \mathrm{~cm}^{-3}$. Careful reading of Fairall's letter shows that he has not used "the mere appearance of the forbidden O III lines" to place a lower limit on the density. There is no such statement of this sort. In implying that $10^{6} \mathrm{~cm}^{-3}$ is an upper limit to the electron density Fairall is merely stating what is basic common knowledge in the field of active galaxy research. It is not normal to spell out pendantically the details of such knowledge every time one writes a research paper (and indeed it would waste valuable journal space to do so).

For my News and Views article ${ }^{2}$ I was asked to explain to the general reader of Nature why Fairall's claim was so remark- able and also to explain such technical terms as "Seyfert 2 galaxy" and "forbidden lines". The explanation I gave of the suppression of forbidden lines at high densities is the standard one that has been given for over half a century. It was first given by Henry Norris Russell in 1927 and is quoted by I. S. Bowen in his 1928 paper on forbidden lines in nebulae ${ }^{3}$. From a pedantic point of view it is, of course, incorrect for us to say that collisions 'prevent' a forbidden transition. A low-probability transition can still be important even at densities orders of magnitude higher than the critical density. This could, for example, be the case for the two-photon $2-1 \mathrm{~s}$ transition in neutral hydrogen in the dense broad-line region clouds of quasars ${ }^{4}$.

I conclude, by stressing that the density and size of the forbidden line regions of Seyfert galaxies and quasars are two of the best known parameters in the subject area.

MARTIN GASKEL
Department of Astronomy,
The University of Texas at Austin.
Austin, Texas 78712 , USA
1. Fairall, A. P. Nature 304, 241-243 (1983).
2. Gaskell, C. M. Nature 304, 212-213 (1983).
3. Bowen, I. S. Astrophys. J. 67, 1-25 (1928).
4. Gaskell, C. M. Observatory 100, 148-153 (1980).

\section{On the half life of ${ }^{138} \mathrm{La}$}

TANAKA AND MASUDA ${ }^{1}$ recently have observed ${ }^{138} \mathrm{Ce} /{ }^{142} \mathrm{Ce}$ isotopic abundance ratios which vary linearly with the ${ }^{138} \mathrm{La} /{ }^{142} \mathrm{Ce}$ abundance ratios in samples of ancient rocks. These variations have been interpreted to be the result of in situ decay of ${ }^{138} \mathrm{La}$ and thus suggest the potential usefulness of the ${ }^{138} \mathrm{La} /{ }^{138} \mathrm{Ce}$ geochronometer. However, to reconcile the age of the samples inferred from the ${ }^{138} \mathrm{La} /{ }^{138} \mathrm{Ce}$ clock with that obtained from the well-established ${ }^{147} \mathrm{Sm} /{ }^{143} \mathrm{Nd}$ chronometer, Tanaka and Masuda had to use a value of the ${ }^{138} \mathrm{La} \beta$-decay partial half life $\sim 16 \%$ less than the mean of the published values for this quantity. To justify the use of this shorter half life, Tanaka and Masuda ${ }^{1}$ argued that the absorption of atmospheric $\mathrm{H}_{2} \mathrm{O}$ and $\mathrm{CO}_{2}$ by $\mathrm{La}_{2} \mathrm{O}_{3}$ probably led to overestimates of the amounts of La contained in samples used in ${ }^{138} \mathrm{La}$ half life determinations.

Recently, however, we have reinvestigated the half life and decay scheme of ${ }^{138} \mathrm{La}$ using $99.999 \%$ pure $\mathrm{La}_{2} \mathrm{O}_{3}$ samples $^{2}$. This material was tested for volatile impurities by the manufacturer (J. T. Baker) by heating it to $1,150^{\circ} \mathrm{C}$ in air for $1 \mathrm{~h}$. Following this procedure, a $0.12 \%$ weight loss was observed. After obtaining 